\title{
Amenable groups and Hadamard spaces with a totally disconnected isometry group
}

\author{
Pierre-Emmanuel Caprace*
}

\begin{abstract}
Let $X$ be a locally compact Hadamard space and $G$ be a totally disconnected group acting continuously, properly and cocompactly on $X$. We show that a closed subgroup of $G$ is amenable if and only if it is (topologically locally finite)-by-(virtually abelian). We are led to consider a set $\partial_{\infty}^{\text {fine }} X$ which is a refinement of the visual boundary $\partial_{\infty} X$. For each $x \in \partial_{\infty}^{\text {fine }} X$, the stabilizer $G_{X}$ is amenable.
\end{abstract}

Mathematics Subject Classification (2000). 20F65; 20E42, 20F50, 43 A07.

Keywords. Amenable group, CAT(0) space, totally disconnected group, locally finite group.

\section{Introduction}

The class of amenable locally compact groups enjoys remarkable closure properties with respect to algebraic operations, such as taking quotients or closed subgroups, or forming group extensions. However, despite of this nice algebraic behaviour, the interaction between the amenability of a given group and its algebraic structure is still not completely understood. This is notably illustrated by the still unresolved problem to show whether or not there exists an infinite finitely generated simple group which is amenable. On the other hand, for some special classes of locally compact groups, the notion of amenability has a very well understood algebraic interpretation. For example, it is known that a connected locally compact group is amenable if and only if its solvable radical is cocompact [Pat88, Theorem 3.8]. Therefore, understanding the structure of amenable locally compact groups amounts to understand the structure of amenable locally compact groups which are totally disconnected. The purpose of this paper is to show that for totally disconnected groups arising in a rather wide geometric context, the algebraic property which is relevant to amenability is the notion of topological local finiteness. A subgroup $H$ of a topological group $G$ is called topologically locally finite if every finite subset of $H$ topologically generates a compact subgroup of $G$. Basic facts on topologically locally finite groups may be

\footnotetext{
${ }^{*}$ F.N.R.S. research fellow, partially supported by the Wiener-Anspach Foundation.
} 
found in Section 2 below. Here we merely mention a result of V. Platonov [Pla65] (see Theorem 2.2 below) which ensures that the class of topologically locally finite groups is closed under group extensions. In particular, any topological group $G$ possesses a topologically locally finite radical, or LF-radical, namely a unique maximal normal subgroup $N$ which is topologically locally finite and such that $G / N$ has no nontrivial normal topologically locally finite subgroup. The LF-radical of $G$ is denoted by $\operatorname{Rad}_{\mathscr{L F}}(G)$.

In this paper we focus on isometry groups of locally compact Hadamard spaces. Recall that a Hadamard space is a complete CAT(0) space. Given a locally compact Hadamard space $X$, its isometry group Is $(X)$, endowed with the topology of uniform convergence on compact subsets, is a locally compact second countable topological group. The main result of this paper is the following:

Theorem 1.1. Let $X$ be a locally compact Hadamard space and $G$ be a totally disconnected group acting continuously, properly and cocompactly on $X$. Then a closed subgroup $H<G$ is amenable if and only if $\operatorname{Rad}_{\mathscr{L} F}(H)$ is open in $H$ and the quotient $H / \operatorname{Rad}_{\mathscr{L F}}(H)$ is virtually abelian.

Note that the hypothesis that $G$ is totally disconnected is naturally satisfied in the case of singular Hadamard spaces, for example when $X$ is a CAT(0) cell complex and the $G$-action is cellular. In fact, the same result as above holds if one replaces the hypothesis that $G$ is totally disconnected and cocompact by the following requirements: $X$ is a locally finite cell complex with finitely many isometry types of cells and finitely many types of links, and the $G$-action is cellular.

Corollary 1.2. Maintain the assumptions of the theorem and let $\Gamma$ be a finitely generated simple subgroup of $G$. The the closure $\bar{\Gamma}$ is amenable if and only if $\Gamma$ is finite. In particular, if $\Gamma$ is amenable, then it is finite.

In the special case when $X$ is $\operatorname{CAT}(-1)$ and $\operatorname{Is}(X)$ acts cocompactly with no fixed point at infinity, one has the following dichotomy: either Is $(X)$ is virtually connected, or it is totally disconnected, see [MMS04, Theorem 21]. This yields the following:

Corollary 1.3. Let $\Gamma$ be a finitely generated infinite simple group acting nontrivially on a CAT(-1) space $X$ such that $\operatorname{Is}(X)$ acts cocompactly with no fixed point at infinity. Then $\Gamma$ has no fixed point in $X \cup \partial_{\infty} X$.

Specializing Theorem 1.1 to discrete groups, we also recover the following result, due to S. Adams and W. Ballmann [AB98, Corollary B]. It is a generalization to amenable subgroups of the so called Solvable Subgroup Theorem for CAT(0) groups [BH99, Theorem II.7.8]: 
Corollary 1.4. Let $\Gamma$ be a group acting properly discontinuously and cocompactly on a complete CAT(0) space $X$. Then any amenable subgroup of $\Gamma$ is virtually abelian and finitely generated.

Applying an argument due to M. Burger and V. Schroeder [BS87, Proof of Corollary 2], one can show furthermore, as in [AB98, Corollary B], that every amenable subgroup of $\Gamma$ leaves some flat of $X$ invariant (the flat is possibly reduced to a point).

We refer to the introduction and reference list of [AB98] for a historical background on amenability in the geometrical context of non-positive curvature. The proof of Theorem 1.1 is based on the one hand, on obstructions for amenable groups to act by isometries on Hadamard spaces established by S. Adams and W. Ballmann [AB98] (see Theorem 5.1 below) and, on the other hand, on an elementary construction which associates to every point $\xi$ of the visual boundary of any CAT(0) space $X$ another CAT( 0$)$ space $X_{\xi}$. This construction is described in Section 4 below; it is originally due to F. Karpelevič [Kar65], who considered it in the context of Riemannian symmetric spaces, (implicitly) by F. Bruhat and J. Tits [BT72, Proposition 7.6.4] in the context of Euclidean buildings, and then by B. Leeb [Lee00, \$2.1.3] in the context of general Hadamard spaces. As suggested in [Lee00], one may iterate this construction to define a boundary $\partial_{\infty}^{\text {fine }} X$ of a proper CAT( 0$)$ space $X$ of bounded geometry which refines the usual visual boundary $\partial_{\infty} X$, in the sense that there is a canonical Is $(X)$-equivariant surjection $\partial_{\infty}^{\text {fine }} X \rightarrow \partial_{\infty} X$. Therefore, the set $\partial_{\infty}^{\text {fine }} X$ is called the refined visual boundary of $X$. A generic point of $\partial_{\infty}^{\text {fine }} X$ is a sequence of the form $\left(\xi_{1}, \xi_{2}, \ldots, \xi_{k}, x\right)$ such that $\xi_{1} \in \partial_{\infty} X, \xi_{i+1} \in \partial_{\infty} X_{\xi_{1}, \ldots, \xi_{i}}$ for each $i=1, \ldots, k-1$ and $x \in X_{\xi_{1}, \ldots, \xi_{k}}$. When $X$ is of bounded geometry, the maximal possible length of this sequence happens to be bounded above by a constant depending only on $X$, see Corollary 4.4 below. The following result provides a more geometric description of amenable subgroups of $G$ :

Theorem 1.5. Let $X$ be a locally compact Hadamard space and $G$ be a totally disconnected group acting continuously, properly and cocompactly on X. Any amenable subgroup of $G$ has a finite index subgroup which fixes a point in $X \cup \partial_{\infty}^{\text {fine }} X$. Conversely, given any point $x \in X \cup \partial_{\infty}^{\text {fine }} X$, the stabilizer $G_{x}$ is amenable.

It is likely that if $\operatorname{Is}(X)$ is cocompact, then the full stabilizer $\operatorname{Is}(X)_{x}$ of any point $x \in X \cup \partial_{\infty}^{\text {fine }} X$ is always amenable. In fact, this is already known if $X$ is CAT(-1) by a result of M. Burger and Sh. Mozes [BM96, Proposition 1.6].

Acknowledgements. A substantial part of this work was accomplished when I was visiting F. Haglund at the Université de Paris XI in Orsay; I thank him for stimulating discussions. The results presented here were first discovered in the special case when $X$ is a building. Their extensions to a higher level of generality benefited from 
exchanges with N. Monod, to whom I express my special gratitude. Finally, I thank A. Lytchak for a careful reading of an earlier version of the manuscript.

\section{On topologically locally finite groups}

Let $G$ be a topological group. A subgroup $H<G$ is called topologically locally finite if the closure of every finitely generated subgroup of $H$ is compact. It is readily seen that if $G$ itself is topologically locally finite, then so is any subgroup and any continuous quotient group. Moreover, we have:

Lemma 2.1. Let $G$ be a locally compact group and $H$ be a topologically locally finite subgroup. Then the closure $\bar{H}$ is topologically locally finite, and $\bar{H}$ endowed with the induced topology is a topologically locally finite group.

Proof. Suppose that $G$ contains a dense subgroup $H$ which is topologically locally finite. We must show that $G$ itself is topologically locally finite. Let $C$ be a relatively compact open neighborhood of the identity in $G$. Given $c_{1}, \ldots, c_{k} \in G$, the subset $C_{1}=\bigcup_{i=0}^{k} c_{i} C$, where $c_{0}=1$, is a relatively compact open neighborhood of the identity containing $c_{1}, \ldots, c_{k}$. We set $U=C_{1} \cup C_{1}^{-1}$. It suffices to prove that the subgroup of $G$ generated by $U$ is compact. Note that this subgroup is open, hence closed.

Let now $y \in \bar{U}^{2}$. Then $y U \cap U^{2}$ is a nonempty open set. Hence there exists $h \in$ $H \cap y U \cap U^{2}$. Since $U=U^{-1}$ and $h \in y U$, we have $y \in h U$. Therefore, we deduce that $\overline{U^{2}} \subset \bigcup_{h \in H \cap U^{2}} h U$. Since $\overline{U^{2}}$ is compact, there exist $h_{1}, \ldots, h_{n} \in H$ such that $\overline{U^{2}} \subset \bigcup_{i=1}^{n} h_{i} U$. Let $K$ be a compact subgroup of $G$ containing $\left\{h_{1}, \ldots, h_{n}\right\}$. Then we have:

$$
U^{3}=U^{2} \cdot U \subset(K \cdot U) \cdot U=K \cdot U^{2} \subset K \cdot K \cdot U=K \cdot U .
$$

We obtain inductively that $U^{n}$ is contained in $K \cdot U$ for each $n$. Since $\langle U\rangle=\bigcup_{n>0} U^{n}$ and since $K \cdot \bar{U}$ is compact, it follows that $\langle U\rangle$ is compact, as desired.

By Zorn's lemma, any topological group $G$ possesses a maximal normal subgroup which is topologically locally finite. It is called the $L F$-radical of $G$ and denoted $\operatorname{Rad}_{\mathscr{L F}}(G)$. By Lemma 2.1, the LF-radical of a locally compact group is a closed subgroup. The following result was proven by V. Platonov [Pla65, Theorem 2]:

Theorem 2.2. Let $G$ be a locally compact group and $N$ be a closed normal subgroup. If $N$ and $G / N$ are both topologically locally finite, then so is $G$.

It follows from Theorem 2.2 that $\operatorname{Rad}_{\mathscr{L F}}\left(G / \operatorname{Rad}_{\mathscr{L F}}(G)\right)=\{1\}$ for any locally compact group $G$. Another useful basic fact is the following: 
Lemma 2.3. Let $G$ be a locally compact group. Then $G$ is topologically locally finite if and only if every compact subset of $G$ is contained in a compact subgroup.

Proof. The 'if' part is clear. We focus on the opposite implication and assume henceforth that $G$ is topologically locally finite. Let $Q$ be a compact subset of $G$ such that $Q=Q^{-1}$. We must show that $Q$ is contained in a compact subgroup of $G$. Up to replacing $G$ by the closed subgroup which is generated by $Q$, this amounts to showing that if $G$ is compactly generated, then it is compact. Let thus $U$ be a compact symmetric neighborhood of the identity which generates $G$. There exist $g_{1}, \ldots, g_{n} \in G$ such that $U^{2} \subset \bigcup_{i=1}^{n} g_{i} U$. Now we can conclude by the same argument as in the proof of Lemma 2.1.

Corollary 2.4. Let $G$ be a locally compact group which is topologically locally finite. Then $G$ is amenable.

Proof. Follows from Lemma 2.3 together with Følner's characterization of amenability.

\section{On proper actions of totally disconnected groups on Hadamard spaces}

Let $X$ be a locally compact Hadamard space, namely a complete locally compact CAT( 0$)$ space. Let also $G$ be a totally disconnected group acting continuously on $X$. Any compact subgroup of $G$ fixes a point in $X$ by [BH99, Chapter II, Corollary 2.8]. Recall that the full isometry group Is $(X)$, endowed with the topology of uniform convergence on compact subsets, is a locally compact second countable group. In particular, if the $G$-action on $X$ is proper, then $G$ is locally compact. The following basic fact will be useful:

Lemma 3.1. Let $G$ be any totally disconnected locally compact group. Then every compact subgroup of $G$ is contained in a compact open subgroup of $G$.

Proof. Let $K$ be a compact subgroup of $G$ and choose a compact open subgroup $Q<G$; it is well-known that such a subgroup exists, see [Bou71, III §4 No 6]. Considering the open cover $\bigcup_{k \in K} k Q$ of the compact group $K$, we may extract finitely many $k_{1}, \ldots, k_{n} \in K$ such that $K \subset \bigcup_{i=1}^{n} k_{n} Q$. In particular $Q$ has finitely many $K$-conjugates and we deduce $Q_{0}:=\bigcap_{k \in K} k Q k^{-1}=\bigcap_{i=1}^{n} k_{n} Q k_{n}^{-1}$. Thus the subgroup $Q_{0}$, which is by definition compact and normalized by $K$, is moreover open. It follows that $K$ is contained in the compact open subgroup $K \cdot Q_{0}$.

We say that the $G$-action is smooth if $G_{x}$ is open in $G$ for each $x \in X$. The term smooth is borrowed from the representation theory of $p$-adic groups. 
The following lemma, though elementary, is crucial to the proof of the main results:

Lemma 3.2. Assume that $G$ acts properly on $X$. Let $\left(x_{n}\right)_{n \geq 0}$ be a sequence of points of $X$ and $\left(\gamma_{n}\right)_{n \geq 0}$ be a sequence of elements of $G$ such that the sequence $\left(\gamma_{n} \cdot x_{n}\right)_{n \geq 0}$ has a subsequence converging to some $c \in X$. Then we have the following:

(i) There exists a sequence $\left(x_{n}^{\prime}\right)_{n \geq 0}$ of points of $X$ such that, given any $g \in G$ with $\lim _{n \rightarrow \infty} d\left(x_{n}, g \cdot x_{n}\right)=0$, we have $g . x_{n}^{\prime}=x_{n}^{\prime}$ for all but a finite number of indices $n \geq 0$.

(ii) Assume moreover that the $G$-action is smooth. Then, given any $g \in G$ such that $\lim _{n \rightarrow \infty} d\left(x_{n}, g . x_{n}\right)=l$, there exists $k \in G$ such that $d(c, k . c)=l$ and that the set $\left\{n \geq 0 \mid \gamma_{n} g \gamma_{n}^{-1} \in k G_{c}\right\}$ is infinite.

Proof. Up to extracting, we may and shall assume that $\lim _{n \rightarrow \infty} \gamma_{n} \cdot x_{n}=c$. Let $g \in G$ be such that $\lim _{n \rightarrow \infty} d\left(x_{n}, g \cdot x_{n}\right)=l$. We have

$$
\lim _{n \rightarrow \infty} d\left(x_{n}, g \cdot x_{n}\right)=\lim _{n \rightarrow \infty} d\left(\gamma_{n} \cdot x_{n},\left(\gamma_{n} g \gamma_{n}^{-1}\right) \gamma_{n} \cdot x_{n}\right)=l .
$$

Therefore, it follows that $\lim _{n \rightarrow \infty} d\left(c, \gamma_{n} g \gamma_{n}^{-1} . c\right)=l$. In particular, the set $\left\{\gamma_{n} g \gamma_{n}^{-1}\right\}_{n \geq 0}$ is relatively compact in $G$. Hence, up to extracting, we may assume that the sequence $\left(\gamma_{n} g \gamma_{n}^{-1}\right)_{n \geq 0}$ converges. By construction, its limit $k$ maps the point $c$ to a point $c^{\prime}$ such that $d\left(c, c^{\prime}\right)=l$.

Assume first that $l=0$. Thus $c=c^{\prime}$. By Lemma 3.1, there exists $x \in X$ such that $G_{c} \subset G_{X}$ and $G_{x}$ is compact open. Since the sequence $\left(\gamma_{n} g \gamma_{n}^{-1}\right)_{n \geq 0}$ converges to $k \in G_{x}$, it follows that the set $\left\{n \geq 0 \mid \gamma_{n} g \gamma_{n}^{-1} \in G_{x}\right\}$ contains all sufficiently large $n$. Now, setting $x_{n}^{\prime}=\gamma_{n}^{-1} . x$, we obtain that $g$ fixes $x_{n}^{\prime}$ for almost all $n$. Thus (i) holds.

Assume now that $l$ is arbitrary and that $G$ acts smoothly. Then $G_{c}$ is open, hence so is the coset $k G_{c}=\left\{h \in G \mid h . c=c^{\prime}\right\}$. Therefore, for all $n$ sufficiently large, we have $\gamma_{n} g \gamma_{n}^{-1} \in k G_{c}$ and (ii) holds.

Recall that, given $\gamma \in G$, the displacement function of $\gamma$ is the map $d_{\gamma}: X \rightarrow \mathbb{R}_{+}$, $x \mapsto d(x, \gamma \cdot x)$. Its infimum is denoted by $|\gamma|$ and is called the translation length of $\gamma$ in $X$.

Note that when $G$ is cocompact, the existence of a sequence $\left(\gamma_{n}\right)_{n \geq 0}$ as in the lemma is automatic. In particular, we obtain (see [BH99, Chapter II, Section 6.16.3]):

Corollary 3.3. Assume that $G$ acts properly and cocompactly on $X$. Then every element $\gamma \in G$ with $|\gamma|=0$ has a fixed point in $X$, and the set $\{|\gamma| \mid \gamma \in G\}$ of translation lengths of elements of $G$ is discrete at 0 . Furthermore, if the $G$-action is smooth, then it is semisimple: any element acts as an elliptic or a hyperbolic isometry. 
Proof. Let $\gamma \in G$ and choose $x_{n} \in X$ so that $d\left(x_{n}, \gamma \cdot x_{n}\right)$ tends to $|\gamma|$ as $n$ tends to infinity. Since $X / G$ is compact, there exists $\gamma_{n} \in G$ such that $\left\{\gamma_{n} . x_{n}\right\}$ is relatively compact in $X$. Thus, up to extracting, we may assume that $\left(\gamma_{n} . x_{n}\right)_{n \geq 0}$ converges to some $c \in X$. If $|\gamma|=0$, then Lemma 3.2 (i) shows that $\gamma_{n} \gamma \gamma_{n}^{-1}$ is elliptic for some $n$, hence so is $\gamma$. Similarly, if the $G$-action is smooth, Lemma 3.2 shows that the displacement function $d_{\gamma}$ attains its infimum $|\gamma|$.

Let now $\left(g_{n}\right)_{n \geq 0}$ be a sequence of elements of $G$ such that $\left|g_{n}\right|$ tends to 0 as $n$ tends to infinity and assume in order to obtain a contradiction that $\left|g_{n}\right|>0$ for all $n$. Since $X / G$ is compact, we may and shall assume, up to replacing $g_{n}$ by a conjugate, that there exists $c \in X, r \in \mathbb{R}$ and $x_{n} \in X$ such that $d\left(c, x_{n}\right)<r$ for all $n$ and that $d\left(x_{n}, g_{n} . x_{n}\right)$ tends to 0 as $n$ tends to infinity. Up to extracting, we may assume that the sequence $\left(x_{n}\right)_{n \geq 0}$ converges to some $x \in X$. Since $\left\{g_{n}\right\}_{n \geq 0}$ is relatively compact in $G$, we may assume, up to a further extraction, that $\left(g_{n}\right)_{n \geq 0}$ converges to some $g \in G$. Clearly $g$ fixes $x$. By Lemma 3.1, this implies that $g_{n}$ is elliptic for all $n$ sufficiently large. Thus $\left|g_{n}\right|=0$, which is absurd.

Recall from [Bri99, Theorem A] that if $X$ is a CAT(0) cell complex with finitely many isometry types of cells, and if the $G$-action is cellular, then it is semisimple and the set of translation lengths of elements of $G$ is discrete at 0 . Thus the hypothesis that $X / G$ is compact is superfluous in that special case. Note that $G$ is automatically smooth in this case.

We record the following observation:

Lemma 3.4. Assume that $G$ acts properly and smoothly on $X$. Let $F \subset X$ be a flat and let $\varphi: G_{\{F\}} \rightarrow \operatorname{Is}(F)$ be the homomorphism induced by the action of the stabilizer $G_{\{F\}}$ of $F$ on $F$. Then $\varphi\left(G_{\{F\}}\right)$ is a discrete subgroup of $\operatorname{Is}(F)$. In particular $G_{\{F\}} / \operatorname{Ker} \varphi$ is virtually abelian and $G_{\{F\}}$ possesses a finite index subgroup which fixes a point in $\partial_{\infty} F$.

Proof. Let $\Gamma=\varphi\left(G_{\{F\}}\right)$. We must show that $\Gamma<\operatorname{Is}(F)$ acts properly discontinuously on $F$.

Let $x \in F$ be any point. We may choose $n+1$ points $x_{0}, \ldots, x_{n}$, where $n=$ $\operatorname{dim} F$, in such a way that the group $G_{\{F\}, x_{0}, \ldots, x_{n}}$ fixes pointwise a neighborhood of $x$ in $F$. Therefore, the group $G_{\{F\}, x_{0}, \ldots, x_{n}}$ is contained in $\operatorname{Ker} \varphi$. Since $G_{x_{0}, \ldots, x_{n}}$ is an open subgroup of the compact open subgroup $G_{x}$, it follows that the index of $G_{x_{0}, \ldots, x_{n}}$ in $G_{x}$ is finite. In particular, for each $x \in F$, the index of $\operatorname{Ker} \varphi$ in $G_{\{F\}, x}$ is finite or, in other words, for each $x \in F$, the stabilizer $\Gamma_{x}$ is finite.

Suppose now that the $\Gamma$-action on $F$ is not properly discontinuous. Then there exist $x_{0} \in F$ and $r \in \mathbb{R}_{+}$such that the set $S_{\Gamma}=\left\{\gamma \in \Gamma \mid d\left(\gamma \cdot x_{0}, x_{0}\right)\right\}$ is infinite. Since $\Gamma_{x}$ is finite for each $x \in X$, it follows that the set $S_{0}=\left\{\gamma \cdot x_{0} \mid \gamma \in S_{\Gamma}\right\}$ is infinite. Let $x_{1} \in F$ be a cluster point of $S_{0}$. Let also $\left(g_{n}\right)_{n \geq 0}$ be a sequence of elements of $G_{\{F\}}$ such that $\lim g_{n} \cdot x_{0}=x_{1}$ and that $g_{m} \cdot x_{0} \neq g_{n} \cdot x_{0}$ for $m \neq n$. 
Since $\left\{g_{n}\right\}_{n \geq 0}$ is relatively compact, we may assume that $\left(g_{n}\right)_{n \geq 0}$ converges to some $g \in G$ such that $g . x_{0}=x_{1}$. Since $G_{x_{0}}$ is open in $G$, so is $g G_{x_{0}}$. Therefore, we have $g_{n} \cdot x_{0}=x_{1}$ for all sufficiently large $n$. This contradicts the fact that $g_{m} . x_{0} \neq g_{n} \cdot x_{0}$ for $m \neq n$. Thus $\Gamma$ is a discrete subgroup of $\operatorname{Is}(F)$.

The fact that $\Gamma$ is virtually abelian now follows from [Thu97, Corollary 4.1.13]. It remains to show that $\Gamma$ has a finite index subgroup which fixes an element in the sphere at infinity $\partial_{\infty} F$. This is trivial if $\Gamma$ is finite. If $\Gamma$ is infinite, then there exists an element $\gamma \in \Gamma$ which acts as a hyperbolic isometry on $F$. Some power of $\gamma$ is centralized by a finite index subgroup $\Gamma_{0}<\Gamma$. Therefore, the group $\Gamma_{0}$ fixes the unique attracting fixed point of $\gamma$ in $\partial_{\infty} F$.

\section{Projective limits of horoballs: the space $X_{\xi}$}

The purpose of this section is to study the main geometrical tool of this paper. In the first subsection, we collect a few subsidiary facts on metric geometry.

4.1. On metric spaces of bounded geometry. Let $(X, d)$ be any metric space. Given $\varepsilon>0$, a subset $N \subset X$ is called $\varepsilon$-sparse if $d\left(x, x^{\prime}\right) \geq \varepsilon$ for all $x \neq x^{\prime} \in N$. Note that a $\varepsilon$-sparse subset is discrete; in particular, if it is contained in a compact subset, then it is finite. Given a subset $C \subset X$, we denote by $n_{\varepsilon}(C)$ the maximal cardinality of a $\varepsilon$-sparse subset of $C$. Note that if $n_{\varepsilon}(C)$ is finite, then a $\varepsilon$-sparse subset $N \subset C$ of maximal possible cardinality is necessarily $\varepsilon$-dense: every point of $C$ is at distance less than $\varepsilon$ from some point of $N$. Given $r>0$ and $\varepsilon>0$, we also set

$$
n_{r, \varepsilon}(X)=\sup _{x \in X} n_{\varepsilon}(B(x, r)),
$$

where $B(x, r)$ denotes the open ball of radius $r$ centered at $x$.

We say that the metric space $(X, d)$ is of bounded geometry if for all $r>\varepsilon>0$, one has $n_{r, \varepsilon}(X)<\infty$. We record some elementary facts for later references:

Lemma 4.1. We have the following:

(i) If $(X, d)$ is complete and of bounded geometry, then it is proper, i.e. any closed ball is compact.

(ii) If $(X, d)$ is locally compact and $X / \operatorname{Is}(X)$ is compact, then $X$ is of bounded geometry.

Proof. (i) follows from the characterization of compact metric spaces as those metric spaces which are complete and totally bounded. The argument goes as follows. Let $B$ be a closed ball in $X$ and $S$ be an infinite set of points of $B$. Since $X$ is of bounded geometry, the ball $B$ can be covered by a finite number of balls of radius 1 . Thus there 
exists $b_{0} \in B$ such that the ball $B\left(b_{0}, 1\right)$ contains an infinite subset of $S$. Repeating this argument inductively, we construct a sequence $\left(b_{n}\right)_{n \geq 0}$ of points of $B$ such that $B\left(b_{n}, 2^{-n}\right)$ contains an infinite subset of $S$ and that $b_{n+1} \in B\left(b_{n}, 2^{-n}\right)$. In particular the sequence $\left(b_{n}\right)_{n \geq 0}$ is Cauchy. Let $b$ denote its limit. Clearly $b$ is a cluster point of $S$. Hence $B$ is compact.

The proof of (ii) is a standard exercise and will be omitted here.

4.2. The space $X_{\xi}$ and the refined boundary $\partial_{\infty}^{\text {fine }} X$. Let $X$ be any CAT(0) space. Given any point $\xi \in \partial_{\infty} X$ in the visual boundary of $X$, we now describe a canonical construction which attaches a CAT(0) space $X_{\xi}$ to $\xi$. Any closed horoball centered at $\xi$ is a closed convex subset of $X$. The collection of all of these horoballs form a chain of subspaces of $X$. Endowing this chain with the orthogonal projections, we obtain a projective system of CAT( 0 ) spaces. By definition, the space $X_{\xi}$ is the metric completion of the projective limit of this system. Note that the projective limit itself need not be complete even if $X$ is so. Indeed, consider for example the convex subset of the Euclidean plane $\mathbb{R}^{2}$ defined by $X=\{(x, y) \mid x y \geq 1, x, y>0\}$. Then the space $X_{\xi}$ associated to the direction of the positive $y$-axis is an open half-line, which is thus not complete. It is therefore important to take a completion since we want to deal with Hadamard spaces. The space $X_{\xi}$ is endowed with a canonical surjective projection

$$
\pi_{\xi}: X \rightarrow X_{\xi}
$$

induced by the orthogonal projections onto horoballs. Note that $\pi_{\xi}$ is 1-Lipschitz: it does not increase distances.

There is a more down-to-earth description of $X_{\xi}$ which goes as follows. Let $X_{\xi}^{*}$ be the set of all geodesics rays of $X$ which point toward $\xi$. The set $X_{\xi}^{*}$ is endowed with a pseudo-distance defined by:

$$
d\left(\rho, \rho^{\prime}\right)=\inf _{t, t^{\prime} \geq 0} d\left(\rho(t), \rho^{\prime}\left(t^{\prime}\right)\right) .
$$

The space $X_{\xi}$ is the completion of the quotient of $X_{\xi}^{*}$ by the relation which identifies two rays at distance 0 , namely two rays which are strongly asymptotic. It is readily verified that this construction yields the same space as the preceding one. Note that $X_{\xi}$ need not be locally compact, even if $X$ is so. Indeed, consider for example the CAT(0) cube complex $X$ which is defined as follows. For each positive integer $n$, we define a closed convex subset $C_{n}$ of the Hilbert space $\ell^{2}(\mathbb{N})$ consisting of those functions $f \in \ell^{2}(\mathbb{N})$ satisfying

$$
\begin{cases}f(0) \in[n-1, n], & \\ f(k) \in[0,1] & \text { for } k \in\{1, \ldots, n-1\}, \\ f(k)=0 & \text { for } k \geq n .\end{cases}
$$


Thus $C_{n}$ is isometric to the unit cube in the Euclidean $n$-space. Now we define $X$ as the union over all $n>0$ of the $C_{n}$ 's, endowed with the length metric induced from $\ell^{2}(\mathbb{N})$ (see [BH99, Definition I.3.3] for the definition of the induced length metric). Thus $X$ is a locally compact CAT( 0$)$ cube complex. Note that the visual boundary $\partial_{\infty} X$ consists of a single point $\xi$, associated with the geodesic ray

$$
\rho: \mathbb{R}_{+} \rightarrow X, \quad t \mapsto t \chi_{\{0\}}
$$

Moreover, it is easily seen that for each $n \geq 0$ the horosphere centered at $\xi$ with base point 0 and radius $-n$ is a $n$-cube. Therefore the space $X_{\xi}$ is an infinite-dimensional cube; in particular it is not locally compact.

The fact that $\pi_{\xi}$ does not increase distances yields the following:

Lemma 4.2. Let $\xi \in \partial_{\infty} X$ and $r, \varepsilon>0$ be positive numbers. Let $x_{0}, x_{1}, \ldots, x_{n} \in$ $X_{\xi}$ be such that $d\left(x_{0}, x_{i}\right)<r$ for each $i$ and that the set $\left\{x_{1}, \ldots, x_{n}\right\}$ is $\varepsilon$-sparse. Then there exist $y_{0}, y_{1}, \ldots, y_{n}, y_{n+1} \in X$ such that $d\left(y_{0}, y_{i}\right)<r$ for each $i$ and that the set $\left\{y_{1}, \ldots, y_{n}, y_{n+1}\right\}$ is $\varepsilon$-sparse.

Proof. Let $\rho_{0}, \rho_{1}, \ldots, \rho_{n}: \mathbb{R}_{+} \rightarrow X$ be geodesic rays which are representatives of $x_{0}, x_{1}, \ldots, x_{n}$ respectively. Note that for all $i=0, \ldots, n$ and $t \in \mathbb{R}_{+}$, we have $\pi_{\xi}\left(\rho_{i}(t)\right)=x_{i}$. Let $R_{0}=\rho_{0}\left(\mathbb{R}_{+}\right)$. By definition, for each $i=1, \ldots, n$ there exists $t_{i} \in \mathbb{R}_{+}$such that $d\left(\rho_{i}\left(t_{i}\right), \operatorname{proj}_{R_{0}}\left(\rho_{i}\left(t_{i}\right)\right)\right)<r$. Here proj denotes the orthogonal CAT(0) projection map [BH99, Chapter II, Proposition 2.4]. Let now $H$ be a closed horoball centered at $\xi$, whose radius is sufficiently small so that $\left\{\rho_{i}\left(t_{i}\right), \operatorname{proj}_{R_{0}}\left(\rho_{i}\left(t_{i}\right)\right) \mid i=1, \ldots, n\right\} \cap H=\varnothing$ and that $\rho_{0}(\varepsilon)$ does not belong to $H$ either. Set $y_{i}=\operatorname{proj}_{H}\left(\rho_{i}(0)\right)$ for each $i=0, \ldots, n$; this makes sense since $H$ is closed and convex. Note that $\operatorname{proj}_{H}\left(\rho_{i}(0)\right)=\operatorname{proj}_{H}\left(\rho_{i}\left(t_{i}\right)\right)$ for all $i>0$. Therefore, we have

$$
d\left(y_{i}, y_{0}\right) \leq d\left(\rho_{i}\left(t_{i}\right), \operatorname{proj}_{R_{0}}\left(\rho_{i}\left(t_{i}\right)\right)\right)<r
$$

for each $i=1, \ldots, n$ since $\operatorname{proj}_{H}$ does not increase distances. Note also that the set $\left\{y_{1}, \ldots, y_{n}\right\}$ is $\varepsilon$-sparse since $\pi_{\xi}$ does not increase distances and since $\left\{x_{1}, \ldots, x_{n}\right\}$ is $\varepsilon$-sparse.

It remains to define $y_{n+1}$. To this end, let $t_{0} \in \mathbb{R}_{+}$be the unique real such that $\rho_{0}\left(t_{0}\right)=y_{0}$. We set $y_{n+1}=\rho_{0}\left(t_{0}-\varepsilon\right)$. Thus $d\left(y_{0}, y_{n+1}\right)=\varepsilon$. Since $\operatorname{proj}_{H}\left(y_{n+1}\right)=y_{0}$, we have $d\left(y, y_{n+1}\right) \geq \varepsilon$ for all $y \in H$. In particular, the set $\left\{y_{1}, \ldots, y_{n}, y_{n+1}\right\}$ is $\varepsilon$-sparse. Finally, since $\varepsilon<r$, we have $d\left(y_{0}, y_{n+1}\right)<r$ as desired.

The following proposition collects some of the basic properties of $X_{\xi}$ :

Proposition 4.3. Let $\xi \in \partial_{\infty} X$. We have the following: 
(i) $X_{\xi}$ is a complete CAT( 0$)$ space.

(ii) There is a canonical continuous homomorphism $\varphi_{\xi}: \operatorname{Is}(X)_{\xi} \rightarrow \operatorname{Is}\left(X_{\xi}\right)$, where $\operatorname{Is}(X)$ and $\operatorname{Is}\left(X_{\xi}\right)$ are endowed with the topology of uniform convergence on compact subsets.

(iii) If $X$ is proper and of bounded geometry, then so is $X_{\xi}$.

Proof. (i) follows immediately from the definition in terms of horoballs. For another argument using the alternative construction of $X_{\xi}$, see B. Leeb [Lee00, Proposition 2.8].

(ii). The map $\varphi_{\xi}$ is defined by

$$
\varphi_{\xi}(g) \cdot \pi_{\xi}(x)=\pi_{\xi}(g \cdot x) .
$$

It is immediate from the definition that it is a homomorphism. Assume in order to obtain a contradiction that $\varphi_{\xi}$ is not continuous. Then it is not continuous at 1 . Thus there exists a compact subset $C \subset X_{\xi}$, a real $\varepsilon>0$, a sequence $\left(y_{n}\right)_{n \geq 0}$ of points of $C$ and a sequence $\left(g_{n}\right)_{n \geq 0}$ of elements of $\operatorname{Is}(X)_{\xi}$ such that $\lim _{n \rightarrow \infty} g_{n}=1$ and $d\left(\varphi_{\xi}\left(g_{n}\right) . y_{n}, y_{n}\right)>\varepsilon$ for each $n$. Let $D \subset C$ be a finite subset which is $\frac{\varepsilon}{3}$-dense in $C$. Let $D^{\prime} \subset X$ be a finite subset such that $\pi_{\xi}\left(D^{\prime}\right)=D$. Since $\lim _{n \rightarrow \infty} g_{n}=1$ and since $D^{\prime}$ is finite, we have $d\left(g_{n} . x, x\right) \leq \frac{\varepsilon}{3}$ for all $x \in D^{\prime}$ and all sufficiently large $n$. Since $\pi_{\xi}$ does not increase distances, we deduce from the definition of $\varphi_{\xi}$ that $d\left(\varphi_{\xi}\left(g_{n}\right) . y, y\right) \leq \frac{\varepsilon}{3}$ for all $y \in D$ and all sufficiently large $n$. Since $D$ is $\frac{\varepsilon}{3}$-dense in $C$, it finally follows that $d\left(\varphi_{\xi}\left(g_{n}\right) . z, z\right) \leq \varepsilon$ for all $z \in C$ and all sufficiently large $n$. This is a contradiction.

Note that $\varphi_{\xi}$ need not be proper.

(iii). By definition, the space $X_{\xi}$ is complete. In view of Lemma 4.1 (i), it is proper whenever it is of bounded geometry. The fact that it is of bounded geometry follows easily from Lemma 4.2.

Important to us will be the fact that the length of a sequence $\left(\xi_{1}, \xi_{2}, \ldots, \xi_{k}\right)$ such that $\xi_{1} \in \partial_{\infty} X$ and $\xi_{i+1} \in \partial_{\infty} X_{\xi_{1}, \ldots, \xi_{i}}$ for each $i=1, \ldots, k-1$ may not be arbitrarily large under suitable assumptions on $X$ :

Corollary 4.4. Let $X$ be a complete CAT(0) space of bounded geometry. Then there exists an integer $K \geq 0$ depending only on $X$ such that, given any sequence $\left(\xi_{1}, \xi_{2}, \ldots, \xi_{k}\right)$ with $\xi_{1} \in \partial_{\infty} X$ and $\xi_{i+1} \in \partial_{\infty} X_{\xi_{1}, \ldots, \xi_{i}}$ for each $i=1, \ldots, k-1$, the space $X_{\xi_{1}, \ldots, \xi_{k}}$ is bounded whenever $k=K$. In particular $\partial_{\infty} X_{\xi_{1}, \ldots, \xi_{k}}$ is empty whenever $k=K$.

Proof. Suppose that $X_{\xi_{1}, \ldots, \xi_{k}}$ is of diameter $>r$. Then $X_{\xi_{1}, \ldots, \xi_{k}}$ contains two points at distance $r$ from one another. Applying Lemma 4.2 inductively, we construct a finite subset $N \subset X$ of cardinality $k+2$ which is $r$-sparse and of radius $\leq r+\varepsilon$, 
where $\varepsilon>0$ is a fixed positive number (which may be chosen arbitrarily small). In particular, we obtain $k+2 \leq n_{r+\varepsilon, r}(X)$. The desired result follows.

Remark. Using results of B. Kleiner [Kle99], it can be shown that if $X$ is complete and $\operatorname{GeomDim}\left(X_{\xi}\right) \geq n$, then $\operatorname{GeomDim}(X) \geq n+1$. In particular, if $\operatorname{GeomDim}(X)$ is finite, then $\operatorname{GeomDim}\left(X_{\xi}\right)<\operatorname{GeomDim}(X)$. Therefore, if $X$ is complete and $\operatorname{GeomDim}(X)$ is finite, then there exists a constant $K$ such that $\partial_{\infty} X_{\xi_{1}, \ldots, \xi_{k}}$ is empty whenever $k \geq K$. Note that a CAT(0) space $X$ such that $\partial_{\infty} X$ is empty might be unbounded: for example take $X$ to be a metric graph which is a star with infinitely many branches of finite length, such that the supremum of the length of the branches is infinite. Note also that the fact that $X$ is of finite geometric dimension is unrelated to the local compactness of $X$. In particular, if $X$ is a CAT(0) piecewise Euclidean cell complex with finitely many types of cells (such as a building [Dav98] or a finite dimensional cube complex), then $\operatorname{GeomDim}(X)$ is finite but $X$ need not be locally compact.

We define the refined visual boundary $\partial_{\infty}^{\text {fine }} X$ to be the set of all sequences

$$
\left(\xi_{1}, \xi_{2}, \ldots, \xi_{k}, x\right)
$$

such that $\xi_{1} \in \partial_{\infty} X, \xi_{i+1} \in \partial_{\infty} X_{\xi_{1}, \ldots, \xi_{i}}$ for each $i=1, \ldots, k-1$ and $x \in X_{\xi_{1}, \ldots, \xi_{k}}$. Given such a sequence $\left(\xi_{1}, \xi_{2}, \ldots, \xi_{k}, x\right)$ in the refined boundary, we define its level to be the number $k$. In order to associate a level to each point of $X \cup \partial_{\infty}^{\text {fine }} X$, we adopt the convention that points of $X$ are of level 0. Corollary 4.4 gives sufficient conditions on $X$ for the existence of an upper bound on the level of all points in $X \cup \partial_{\infty}^{\text {fine }} X$.

4.3. Structure of the stabilizer of a point in the refined boundary. Given a point $\xi \in \partial_{\infty} X$ and a base point $x \in X$, we let $b_{\xi, x}: X \rightarrow \mathbb{R}$ be the Busemann function centered at $\xi$ such that $b_{\xi, x}(x)=0$. Recall that Busemann functions satisfy the following cocycle identity for all $x, y, z \in X$ :

$$
b_{\xi, x}(y)-b_{\xi, x}(z)=b_{\xi, z}(y) .
$$

It follows that the mapping

$$
\beta_{\xi}: \operatorname{Is}(X)_{\xi} \rightarrow \mathbb{R}, \quad g \mapsto b_{\xi, x}(g \cdot x),
$$

is independent of the point $x \in X$ and is a group homomorphism. It is called the Busemann homomorphism centered at $\xi$.

Proposition 4.5. Let $X$ be a proper $\mathrm{CAT}(0)$ space and $G$ be a totally disconnected group acting continuously, properly and cocompactly on $X$. Given $\xi \in \partial_{\infty} X$, we have the following: 
(i) Given any $x \in X_{\xi}$, the $L F$-radical $\operatorname{Rad}_{\mathscr{L} F}\left(G_{\xi, x}\right)$ is open in $G_{\xi, x}$; it coincides with the kernel of $\beta_{\xi}: G_{\xi, x} \rightarrow \mathbb{R}$.

(ii) Let $K_{\xi}$ be the kernel of the restriction of $\varphi_{\xi}$ to $G_{\xi}$, where $\phi_{\xi}$ is defined by (4.1) on page 447. Then $\operatorname{Rad}_{\mathscr{L F}}\left(K_{\xi}\right)$ is open in $K_{\xi}$; it coincides with the kernel of $\beta_{\xi}: K_{\xi} \rightarrow \mathbb{R}$. In particular, the group $K_{\xi} / \operatorname{Rad}_{\mathscr{L}}\left(K_{\xi}\right)$ is isomorphic to a subgroup of $\mathbb{R}$.

(iii) Let $\left(\xi_{1}, \xi_{2}, \ldots, \xi_{n}, x\right) \in \partial_{\infty}^{\mathrm{fine}} X$ be a point of level $n$ in the refined visual boundary. Set $H=G_{\xi_{1}, \ldots, \xi_{n}, x}$. Then $\operatorname{Rad}_{\mathscr{L} \mathscr{F}}(H)$ is open in $H$, it contains all elements of $H$ which act as elliptic isometries on $X$ and, furthermore, $H / \operatorname{Rad}_{\mathcal{L F}}(H)$ is abelian and torsion free. In particular $H$ is amenable.

Proof. Note that (i) is a special case of (iii). However, the proof of (iii) involves some technicalities which can be avoided in the situation of (i). Therefore, in order to make the argument more transparent, we prove (i) separately.

(i). Let $K_{\xi, x}$ denote the kernel of the restriction to $G_{\xi, x}$ of the Busemann homomorphism $\beta_{\xi}$. Let $y \in X$ be such that $\pi_{\xi}(y)=x$ and $\rho=\rho_{\xi, y}: \mathbb{R}_{+} \rightarrow X$ be the geodesic ray pointing towards $\xi$ with origin $y$. Define $x_{n}=\rho(n)$ for each $n \in \mathbb{N}$. Since $G$ is cocompact, there exists a sequence $\left(\gamma_{n}\right)_{n \geq 0}$ of elements of $G$ such that $\left(\gamma_{n} \cdot x_{n}\right)_{n \geq 0}$ converges to some $c \in X$. Now, given any $g_{1}, \ldots, g_{k} \in K_{\xi, x}$, we have $\lim _{n \rightarrow \infty} d\left(x_{n}, g_{i} . x_{n}\right)=0$ for each $i=1, \ldots, k$. Therefore, applying Lemma 3.2 inductively, we deduce that there exists $n \in \mathbb{N}$ such that $g_{i} \in G_{\gamma_{n}^{-1} . c}$ for each $i=1, \ldots, k$. In particular, the set $\left\{g_{1}, \ldots, g_{k}\right\}$ is contained in a compact subgroup of $G$. This shows that $K_{\xi, x}$ is topologically locally finite.

Now, the inclusion $K_{\xi, x} \subset \operatorname{Rad}_{\mathscr{L} F}\left(G_{\xi, x}\right)$ is obvious. Conversely, given any element $g \in G_{\xi, x}$ which does not belong to $K_{\xi, x}$, then $g$ is not elliptic, hence it is not contained in $\operatorname{Rad}_{\mathscr{L} \mathcal{F}}\left(G_{\xi, x}\right)$. Thus $K_{\xi, x}=\operatorname{Rad}_{\mathscr{L} \mathcal{F}}\left(G_{\xi, x}\right)$ as desired.

The fact that $K_{\xi, x}$ is open in $G_{\xi, x}$ is clear: by definition $G_{\xi, x}$ is closed and any compact open subgroup of $G_{\xi, x}$ fixes a point in $X$, and is thus contained in $K_{\xi, x}$.

(ii). By definition, we have $K_{\xi}=\bigcap_{x \in X_{\xi}} G_{\xi, x}$. Hence the desired assertion follows from (i).

(iii). For each $i=1, \ldots, n$, let $\beta_{\xi_{i}}: G_{\xi_{1}, \ldots, \xi_{i}} \rightarrow \mathbb{R}$ be the restriction of the Busemann homomorphism centered at $\xi_{i}$. In particular, restricting further, one obtains a homomorphism $\beta_{\xi_{i}}: H \rightarrow \mathbb{R}$. The direct product of these homomorphisms defines a homomorphism

$$
\beta=\beta_{\xi_{1}} \times \cdots \times \beta_{\xi_{n}}: H \rightarrow \mathbb{R}^{n},
$$

whose kernel is the subgroup $K=\bigcap_{i=1}^{n} \operatorname{Ker} \beta_{\xi_{i}}$. Clearly $K$ contains all elements of $H$ which act as elliptic isometries on $X$ (and hence on $X_{\xi_{1}, \ldots, \xi_{i}}$ for each $\left.i=1, \ldots, n\right)$. In particular it follows that $K$ is open in $H$.

Our aim is to show that $K=\operatorname{Rad}_{\mathscr{L} \mathcal{F}}(H)$. We have just seen that $K$ contains all periodic elements of $H$. Thus the inclusion $\operatorname{Rad}_{\mathscr{L} \mathcal{F}}(H) \subset K$ is clear. It remains to show that $K$ is topologically locally finite. 
For each $i=1, \ldots, n$, we define

$$
\varphi_{i}=\varphi_{\xi_{i}} \circ \ldots \circ \varphi_{\xi_{1}} \circ \varphi_{\xi_{1}}: \operatorname{Is}(X)_{\xi_{1}, \ldots, \xi_{i}} \rightarrow \operatorname{Is}\left(X_{\xi_{1}, \ldots, \xi_{i}}\right) .
$$

Let $g_{1}, \ldots, g_{k}$ be elements of $K$. By definition, there exists a sequence $\left(x_{n-1, m}\right)_{m \geq 0}$ of points of $X_{\xi_{1}, \ldots, \xi_{n-1}}$ such that

$$
\lim _{m \rightarrow \infty} d\left(\varphi_{n-1}\left(g_{i}\right) \cdot x_{n-1, m}, x_{n-1, m}\right)=0
$$

for each $i=1, \ldots, k$. Let now $\rho_{n-2, m}: \mathbb{R}_{+} \rightarrow X_{\xi_{1}, \ldots, \xi_{n-2}}$ be a geodesic ray pointing towards $\xi_{n-1}$ such that $\pi_{\xi_{n-1}}\left(\rho_{n-2, m}(t)\right)=x_{n-1, m}$ for each $t \in \mathbb{R}_{+}$.

For each $m$, we may choose a sufficiently large $t_{m} \in \mathbb{R}_{+}$in such a way that the sequence $\left(x_{n-2, m}\right)_{m \geq 0}$ defined by $x_{n-2, m}=\rho_{n-2, m}\left(t_{m}\right) \in X_{\xi_{1}, \ldots, \xi_{n-2}}$ satisfies the identity

$$
\lim _{m \rightarrow \infty} d\left(\varphi_{n-2}\left(g_{i}\right) \cdot x_{n-2, m}, x_{n-2, m}\right)=0
$$

for each $i=1, \ldots, k$.

Proceeding inductively, we construct in this way a sequence $\left(x_{j, m}\right)_{m \geq 0}$ of points of $X_{\xi_{0}, \ldots, \xi_{j}}$ such that

$$
\lim _{m \rightarrow \infty} d\left(\varphi_{j}\left(g_{i}\right) \cdot x_{j, m}, x_{j, m}\right)=0
$$

for each $i=1, \ldots, k$ and each $j=1, \ldots, n-1$. In a final further step, we then construct a sequence $\left(x_{m}\right)_{m \geq 0}$ of points of $X$ such that

$$
\lim _{m \rightarrow \infty} d\left(g_{i} \cdot x_{m}, x_{m}\right)=0
$$

for each $i=1, \ldots, k$. Now, it follows by the same arguments as in the proof of (i) that $\left\{g_{1}, \ldots, g_{k}\right\}$ is contained in a compact subgroup of $G$. Hence $K$ is topologically locally finite, as desired.

The amenability of $H$ is now immediate from Corollary 2.4.

Note that the proof of Proposition 4.5 (iii) shows that $\operatorname{Rad}_{\mathscr{L} F}(H)$ coincides with $\left.\operatorname{Ker} \beta\right|_{H}$, where $\beta=\beta_{\xi_{1}} \times \cdots \times \beta_{\xi_{n}}: G_{\xi_{1}, \ldots, \xi_{n}} \rightarrow \mathbb{R}^{n}$ is the direct product of the Busemann homomorphisms centered at $\xi_{i}$ for $i=1, \ldots, n$.

Lemma 4.6. Let $X$ be a proper CAT(0) space and $G$ be a totally disconnected group acting continuously, properly and cocompactly on $X$. Then, given any element $\gamma \in \operatorname{Ker} \beta$, the respective translation lengths of $\gamma$ in $X$ and in $X_{\xi_{1}, \ldots, \xi_{n}}$ coincide. Furthermore, if the $G$-action is smooth, then the action of $G_{\xi_{1}, \ldots, \xi_{n}}$ on $X_{\xi_{1}, \ldots, \xi_{n}}$ is by semisimple isometries.

Proof. Let $\xi \in \partial_{\infty} X$. Since $\pi_{\xi}$ does not increase distances, it is clear that the translation length $|\gamma|$ of any element $\gamma \in \operatorname{Is}(X)_{\xi}$ is bounded below by the translation 
length $\left|\varphi_{\xi}(\gamma)\right|$ of $\varphi_{\xi}(\gamma)$ in $X_{\xi}$. Conversely, if $\gamma \in \operatorname{Ker} \beta$, then it is easy to see that $|\gamma| \leq\left|\varphi_{\xi}(\gamma)\right|$.

It is clear that an elliptic isometry $\gamma \in \operatorname{Is}(X)$ which fixes $\xi$ acts as an elliptic isometry on $X_{\xi}$. Suppose now that $\gamma \in \operatorname{Is}(X)$ is hyperbolic and fixes $\xi$. Let $\lambda$ be an axis of $\gamma$. If $\xi \in \partial_{\infty} \lambda$, then $\gamma$ is elliptic on $X_{\xi}$. Otherwise, it follows easily from [BH99, Chapter II, Proposition 9.8 and Corollary 9.9] that $\lambda$ bounds a Euclidean halfplane $H$ such that $\xi \in \partial_{\infty} H$. Moreover, one verifies immediately that the projection of $H$ to $X_{\xi}$ is an axis for $\gamma$, from which it follows that $\gamma$ acts as a hyperbolic isometry on $X_{\xi}$. Note moreover that $\beta_{\xi}(\gamma)=0$ if and only if $\xi$ is the middle point of $\partial_{\infty} H$, where $\beta_{\xi}$ denotes the Busemann homomorphism centered at $\xi$.

Now, if the $G$-action is smooth, the fact that the $G_{\xi_{1}, \ldots, \xi_{n}}$-action on $X_{\xi_{1}, \ldots, \xi_{n}}$ is semisimple follows from a straightforward induction on $n$, since we know by Corollary 3.3 that the $G$-action on $X$ is semisimple.

\section{The structure of amenable subgroups}

The main tool in proving Theorem 1.1 is provided by the obstructions for continuous isometric actions of amenable groups on locally compact Hadamard spaces established by S. Adams and W. Ballmann in [AB98]. Let us recall its precise statement:

Theorem 5.1. Let $H$ be an amenable locally compact group acting continuously by isometries on a proper CAT( 0$)$ space $X$. Then $H$ stabilizes a Euclidean flat in $X$, or else $H$ fixes a point in $X \cup \partial_{\infty} X$.

Proof. See [AB98, Theorem].

Before proceeding to the proof of the main results, we still need a subsidiary lemma:

Lemma 5.2. Let $X$ be a proper CAT(0) space and $G$ be a totally disconnected group acting continuously, properly and cocompactly on $X$. Let $\left(\xi_{1}, \ldots, \xi_{n}\right)$ be a sequence such that $\xi_{1} \in \partial_{\infty} X, \xi_{i+1} \in \partial_{\infty} X_{\xi_{1}, \ldots, \xi_{i}}$ for each $i=1, \ldots, n-1$ and let $F$ be aflat in $X_{\xi_{1}, \ldots, \xi_{n}}$ (possibly $n=0$ and $F \subset X$ ). Suppose that $H<G$ is a closed amenable subgroup which fixes $\left(\xi_{1}, \ldots, \xi_{n}\right)$ and which stabilizes $F$. Then $H$ possesses a finite index subgroup which fixes a point in $F \cup \partial_{\infty} F$.

Proof. As in the proof of Proposition 4.5 (iii), we let $\beta_{\xi_{i}}: G_{\xi_{1}, \ldots, \xi_{i}} \rightarrow \mathbb{R}$ be the restriction of the Busemann homomorphism centered at $\xi_{i}$ and

$$
\beta=\beta_{\xi_{1}} \times \cdots \times \beta_{\xi_{n}}: G_{\xi_{1}, \ldots, \xi_{n}} \rightarrow \mathbb{R}^{n}
$$

be the direct product of these Busemann homomorphisms. Let $R=\operatorname{Ker} \beta$. 
By hypothesis, we have $H<\operatorname{Stab}_{G_{\xi_{1}, \ldots, \xi n}}(F)$. Thus there is a well defined homomorphism

$$
\varphi: H \rightarrow \operatorname{Is}(F) .
$$

Since $H$ is totally disconnected, it follows from [MZ55, Chapter V, Theorem 2] that $\varphi(H)$ (endowed with the quotient topology) is a discrete group. Since moreover $\varphi(H)$ is amenable and contained in the real Lie group Is $(F)$, it follows from [Tit72, Theorem 1] that $\varphi(H)$ is virtually solvable, hence virtually metabelian because $\operatorname{Is}(F)$ is abelian-by-compact. Up to replacing $H$ by a finite index subgroup, we may - and shall - assume henceforth that $\varphi(H)$ is metabelian.

Let $T$ denote the translation subgroup of $\operatorname{Is}(F)$. Thus we have $[\varphi(H), \varphi(H)] \subset T$. On the other hand, since $R=\operatorname{Ker} \beta$ contains the derived group $[H, H]$, we deduce that $[\varphi(H), \varphi(H)] \subset T \cap \varphi(H \cap R)$. Now we distinguish several cases.

Assume first that $T \cap \varphi(H \cap R)$ is nontrivial. By Corollary 3.3 and Lemma 4.6, the set of translation lengths of elements of $R$ in $X_{\xi_{1}, \ldots, \xi_{n}}$ is discrete at 0 . Therefore, it follows that $T \cap \varphi(H \cap R)$ is a discrete subgroup of $T$. Let now $t \in T \cap \varphi(H \cap R)$ be a nontrivial element. Since $T \cap \varphi(H \cap R)$ is normal in $\varphi(H)$ and since conjugate elements act with the same translation length, it follows from the discreteness of $T \cap \varphi(H \cap R)$ in $T$ that $\varphi(H)$ possesses a finite index subgroup which centralizes $t$. Since $t$ acts as a hyperbolic element, we deduce that its unique attractive fixed point in the sphere at infinity $\partial_{\infty} F$ is fixed by a finite index subgroup of $H$. Hence we are done in this case.

We assume henceforth that $T \cap \varphi(H \cap R)$ is trivial. By the above, it follows that $\varphi(H)$ is abelian. Suppose now $\varphi(H)$ contains an element $t^{\prime}$ which acts as a hyperbolic element on $F$. Then $\varphi(H)$ fixes the attractive fixed point of $t^{\prime}$ in $\partial_{\infty} F$ and again we are done. Suppose finally that every element in $\varphi(H)$ is elliptic. Since the fixed point set of an element in $\operatorname{Is}(F)$ is a linear, hence Euclidean, subspace, a straightforward induction on dimension shows then that $\varphi(H)$ has a global fixed point in $F$. This concludes the proof.

We are now ready for the

Proof of Theorems 1.1 and 1.5. Note that $X$ is complete and of bounded geometry, since $\operatorname{Is}(X)$ is cocompact by hypothesis.

The fact that $G_{X}$ is (topologically locally finite)-by-(virtually abelian) for each $x \in X \cup \partial_{\infty}^{\text {fine }} X$ follows from Proposition 4.5 (iii). Any such subgroup is amenable in view of Corollary 2.4 .

Let now $H<G$ be a closed amenable subgroup. We want to show that $H$ possesses a finite index subgroup which fixes an element of $X \cup \partial_{\infty}^{\text {fine }} X$.

Assume that $H$ fixes no point in $X \cup \partial_{\infty} X$. In view of Theorem 5.1, this implies that $H$ stabilizes a flat $F \subset X$. By Lemma 5.2, we deduce that $H$ possesses a finite 
index subgroup which fixes a point in $\partial_{\infty} F$. This shows that in all cases $H$ possesses a finite index subgroup $H_{0}$ which fixes a point $\xi_{1} \in X \cup \partial_{\infty} X$.

If $\xi_{1} \in X$ we are done. Otherwise $H_{0}$ acts on $X_{\xi_{1}}$. Assume that $H_{0}$ fixes no point in $X_{\xi_{1}} \cup \partial_{\infty} X_{\xi_{1}}$. Then $H_{0}$ stabilizes a flat in $X_{\xi_{1}}$ and, by Lemma 5.2, we deduce that $H_{0}$ possesses a finite index subgroup $H_{1}$ which fixes a point $\xi_{2}$ in $X_{\xi_{1}} \cup \partial_{\infty} X_{\xi_{1}}$. Again, if $\xi_{2} \in X_{\xi_{1}}$ we are done. Otherwise $H_{2}$ acts on $X_{\xi_{1}}, \xi_{2}$.

Now we repeat this argument inductively. The process will stop after finitely many steps in view of Corollary 4.4. Therefore, we obtain a point $\left(\xi_{1}, \ldots, \xi_{n}, x\right) \in \partial_{\infty}^{\text {fine }} X$ and a closed subgroup $H_{n}<H$ of finite index which is contained in $G_{\xi_{1}, \ldots, \xi_{n}, x}$. By Proposition 4.5 (iii), the latter subgroup is (topologically locally finite)-by-(abelian torsion free) and its LF-radical is open.

Proof of Corollary 1.2. Let $\Gamma<G$ be a finitely generated simple subgroup which is contained in an amenable subgroup of $G$. In view of the characterization of amenability in terms of a fixed point property [BdlHV07, Theorem G.1.7], we may and shall assume that $\Gamma$ is in fact contained in a closed amenable subgroup of $G$, say $H$. Let $H_{0}$ be its LF-radical. There are two cases.

Suppose first that $H_{0} \cap \Gamma$ is trivial. Then $\Gamma$ injects in the quotient $H / H_{0}$, which is virtually abelian. Since $\Gamma$ is simple and finitely generated, it must then be finite.

Suppose now that $H_{0} \cap \Gamma$ is nontrivial. Then $\Gamma \subset H_{0}$. Therefore $\Gamma$ is contained in a compact subgroup of $G$. Since any such subgroup is a profinite group, it follows that $\Gamma$ is residually finite. Hence, since $\Gamma$ is simple, it must be finite.

Proof of Corollary 1.3. By [MMS04, Theorem 21], the group Is $(X)$ is either virtually connected or totally disconnected. By assumption Is $(X)$ contains a finitely generated infinite simple group $\Gamma$. Such a group cannot be contained in a connected locally compact group. Indeed, a connected locally compact group is an extension of a Lie group by a compact group [MZ55], and any finitely generated subgroup of a compact group (resp. a Lie group) is residually finite. Thus we may assume without loss of generality that $\operatorname{Is}(X)$ is totally disconnected. Now, given any $x \in X$, the stabilizer Is $(X)_{x}$ is compact and, hence, cannot contain the simple group $\Gamma$. Finally, given any $\xi \in \partial_{\infty} X$, the stabilizer Is $(X)_{\xi}$ is amenable [BM96, Proposition 1.6] and the conclusion now follows from Corollary 1.2.

Proof of Corollary 1.4. Let $H<\Gamma$ be an amenable subgroup. Let $F$ be its LFradical. It is a discrete countable locally finite group. In particular, it is a union of an ascending chain of finite subgroups of $\Gamma$. Since $\Gamma$ acts geometrically on $X$, it follows from [BH99, Chapter II, Corollary 2.8] that it has finitely many conjugacy classes of finite subgroups. In particular $F$ is finite. Therefore, there exists a finite index subgroup $H_{0}<H$ which centralizes $F$. By Theorem 1.1, the group $H / F$ is virtually abelian. Thus $H_{0}$ possesses a finite index subgroup $H_{1}$ such that the derived subgroup $\left[H_{1}, H_{1}\right]$ is contained in $F$. Since any finitely generated group with a finite 
derived subgroup is virtually abelian [BH99, Chapter II, Lemma 7.9], it follows that any finitely generated subgroup of $H_{1}$ is virtually abelian. On the other hand, the group $\Gamma$ satisfies an ascending chain condition for virtually abelian subgroups by [BH99, Chapter II, Theorem 7.5], from which it finally follows that $H_{1}$ is virtually abelian and finitely generated and, hence, so is $H$.

\section{References}

[AB98] S. Adams and W. Ballmann, Amenable isometry groups of Hadamard spaces. Math. Ann. 312 (1998), 183-195. Zbl 0913.53012 MR 1645958

[BdlHV07] B. Bekka, P. de la Harpe, and A. Valette, Kazhdan's property (T). New Math. Monogr. 11, Cambridge University Press, Cambridge 2008. Zbl 1146.22009 MR 2415834

[BH99] M. R. Bridson and A. Haefliger, Metric spaces of non-positive curvature. Grundlehren Math. Wiss. 319, Springer-Verlag, Berlin 1999. Zbl 0988.53001 MR 1744486

[BM96] M. Burger and Sh. Mozes, CAT(-1)-spaces, divergence groups and their commensurators. J. Amer. Math. Soc. 9 (1996), 57-93. Zbl 0847.22004 MR 1325797

[Bou71] N. Bourbaki, Éléments de mathématique. Topologie générale. Chapitres 1 à 4. Hermann, Paris 1971. Zbl 0249.54001 MR 0358652

[Bri99] M. Bridson, On the semisimplicity of polyhedral isometries. Proc. Amer. Math. Soc. 127 (1999), 2143-2146. Zbl 0928.52007 MR 1646316

[BS87] M. Burger and V. Schroeder, Amenable groups and stabilizers of measures on the boundary of a Hadamard manifold. Math. Ann. 276 (1987), 505-514. Zbl 0599.53036 MR 875344

[BT72] F. Bruhat and J. Tits, Groupes réductifs sur un corps local. I: Données radicielles valuées. Inst. Hautes Études Sci. Publ. Math. 41 (1972), 5-251. Zbl 0254.14017 MR 0327923

[Dav98] M. Davis, Buildings are CAT(0). In Geometry and cohomology in group theory (Durham, 1994) (Cambridge), H. Kropholler et al., eds., London Math. Soc. Lecture Note Ser. 252, Cambridge University Press, Cambridge 1998, 108-123. Zbl 0978.51005 MR 1709955

[Kar65] F. I. Karpelevich, The geometry of geodesics and the eigenfunctions of the Beltrami-Laplace operator on symmetric spaces. Trudy Moskov. Mat. Ob̌̌c. 14 (1965), 48-185; English transl. Trans. Mosc. Math. Soc. 14 (1965), 51-199. Zbl 0164.22202 MR 0231321

[Kle99] B. Kleiner, The local structure of length spaces with curvature bounded above. Math. Z. 231 (1999), 409-456. Zbl 0940.53024 MR 1704987

[Lee00] B. Leeb, A characterization of irreducible symmetric spaces and Euclidean buildings of higher rank by their asymptotic geometry. Bonn. Math. Schr. 326, Universität Bonn, Mathematisches Institut, Bonn 2000. Zbl 1005.53031 MR 1934160 
[MMS04] I. Mineyev, N. Monod, and Y. Shalom, Ideal bicombings for hyperbolic groups and applications. Topology 43 (2004), 1319-1344. Zbl 1137.20033 MR 2081428

[MZ55] D. Montgomery and L. Zippin, Topological transformation groups. Interscience Publishers, New York, London 1955. Zbl 0068.01904 MR 0073104

[Pat88] A. L. T. Paterson, Amenability. Math. Surveys Monogr. 29, Amer. Math. Soc., Providence, RI, 1988. Zbl 0648.43001 MR 961261

[Pla65] V. P. Platonov, Lokal projective nilpotent radicals in topological groups. Dokl. Akad. Nauk BSSR 9 (1965), 573-577 (in Russian). Zbl 0242.22006

[Thu97] W. P. Thurston, Three-dimensional geometry and topology. Vol. 1 (Silvio Levy, ed.), Princeton Math. Ser. 35, Princeton University Press, Princeton, NJ, 1997. Zbl 0873.57001 MR 1435975

[Tit72] J. Tits, Free subgroups in linear groups. J. Algebra 20 (1972), 250-270. Zbl 0236.20032 MR 0286898

Received June 12, 2007

Pierre-Emmanuel Caprace, Département de Mathématiques, Université catholique de Louvain, Chemin du Cyclotron, 2, 1348 Louvain-la-Neuve, Belgique

E-mail: pe.caprace@uclouvain.be 